\title{
Incommensurate-commensurate transitions in the monoaxial chiral helimagnet driven by the magnetic field
}

\author{
Victor Laliena, ${ }^{1, *}$ Javier Campo, ${ }^{1, \dagger}$ Jun-Ichiro Kishine, ${ }^{2,3}$ Alexander S. Ovchinnikov, ${ }^{4}$ Yoshihiko Togawa, ${ }^{3,5}$ \\ Yusuke Kousaka, ${ }^{3,6}$ and Katsuya Inoue ${ }^{3,6}$ \\ ${ }^{1}$ Instituto de Ciencia de Materiales de Aragón (CSIC_University of Zaragoza), C/Pedro Cerbuna 12, 50009 Zaragoza, Spain \\ ${ }^{2}$ Division of Natural and Environmental Sciences, The Open University of Japan, Chiba, 261-8586, Japan \\ ${ }^{3}$ Centre for Chiral Science, Hiroshima University, Higashi-Hiroshima, Hiroshima 739-8526, Japan \\ ${ }^{4}$ Institute of Natural Sciences, Ural Federal University, Ekaterinburg 620083, Russia \\ ${ }^{5}$ N2RC, Osaka Prefecture University, 1-2 Gakuencho, Sakai, Osaka 599-8570, Japan \\ ${ }^{6}$ Department of Chemistry, Faculty of Science, Hiroshima University, Higashi-Hiroshima, Hiroshima 739-8526, Japan
}

(Received 28 August 2015; revised manuscript received 25 February 2016; published 20 April 2016)

\begin{abstract}
The zero-temperature phase diagram of the monoaxial chiral helimagnet in the magnetic-field plane formed by the components parallel and perpendicular to the helical axis is thoroughly analyzed. The nature of the transition to the commensurate state depends on the angle between the field and the helical axis. For field directions close to the directions parallel or perpendicular to the helical axis the transition is continuous, while for intermediate angles the transition is discontinuous and the incommensurate and commensurate states coexist on the transition line. The continuous and discontinuous transition lines are separated by two tricritical points with specific singular behavior. The location of the continuous and discontinuous lines and of the tricritical points depend strongly on the easy-plane anisotropy, the effect of which is analyzed. For high anisotropy the conical approximation locates the transition line very accurately, although it does not predict the continuous transitions and the tricritical behavior. It is shown that for high anisotropy, as in $\mathrm{CrNb}_{3} \mathrm{~S}_{6}$, the form of the transition line is universal, that is, independent of the sample, and obeys a simple equation. The position of the tricritical points, which is not universal, is theoretically estimated for a sample of $\mathrm{CrNb}_{3} \mathrm{~S}_{6}$.
\end{abstract}

DOI: 10.1103/PhysRevB.93.134424

\section{INTRODUCTION}

Chiral magnets are very promising ingredients for spintronic-based devices since they support peculiar magnetic textures that affect the charge and spin transport properties in different ways [1]. As these magnetic textures can be deeply altered by magnetic fields, the transport properties can be magnetically controlled, and thus they might be used as functional components of magnetic devices [2,3].

For example, in the monoaxial chiral helimagnet, an example of which is $\mathrm{CrNb}_{3} \mathrm{~S}_{6}$, the competition between ferromagnetic (FM) and Dzyaloshinskii-Moriya (DM) interactions leads to an incommensurate magnetic helix propagating with period $L_{0}$ along a crystallographic axis, which is called here the DM axis. The effect of the magnetic field on the helical magnetic ordering is an old topic and was discussed, for instance, in Ref. [4]. In the case of monoaxial DM interactions, application of a magnetic field deforms the helix in a way which depends on the field direction. If the field is parallel to the DM axis, a conical helix is formed, with the spins tilted by a constant angle towards the DM axis, while revolving around it with a period $L_{0}$ independent of the magnetic field. If, on the other hand, the magnetic field is perpendicular to the DM axis, the spins remain perpendicular to the DM axis but tend to be aligned with the magnetic field. The spins rotate slowly about the DM axis in the regions where they are nearly parallel to the magnetic field, and the rotation becomes faster as the spin direction separates from the field direction. As

\footnotetext{
*laliena@unizar.es

†javier.campo@csic.es
}

the field increases, the regions where the spins rotate slowly become very wide and the regions where the spins rotate rapidly become very narrow, and a chiral soliton lattice (CSL) is formed [1,5-7]. This CSL, which is realized in $\mathrm{CrNb}_{3} \mathrm{~S}_{6}$ [8], supports dynamic modes like coherent sliding [9] and gives rise to exciting phenomena such as spin motive forces [10] and tunable magnetoresistance [11-14].

If the applied field is strong enough, the incommensurate chiral helicoid is transformed into a commensurate FM state, called the forced ferromagnetic (FFM) state. Chiral and incommensurate (IC)-to-commensurate (C) transitions, which are universal and appear in many branches of physics, take place in these systems. The nature of the IC-C transition and its driving mechanism depends on the angle $\alpha$ between the field and the DM axis. If a field parallel to the DM axis increases, the cone on which the spins lie becomes narrower, but the helix period $L_{0}$ remains constant. At a certain critical field the cone closes completely and the FFM state is reached continuously. On the other hand, the effect of increasing a field perpendicular to the DM axis is to increase the period of the CSL, so that the FFM state is reached continuously in a critical field at which the period $L$ of the CSL diverges [1]. Thus, in the two limiting cases of magnetic fields parallel and perpendicular to the DM axis the FFM state is reached via two very different mechanisms. On the other hand, even though these systems have been extensively studied, the nature of the transition to the FFM has not been elucidated yet when $\alpha$ is continuously varied between the limiting cases 0 and $\pi / 2$.

A theoretical attempt has been made recently based on an approximation which makes the model equations easily solvable [15]. As we show in Sec. VI the approximation gives the transition line very accurately when the easy-plane 
anisotropy is high, as in the case of $\mathrm{CrNb}_{3} \mathrm{~S}_{6}$. However, the nature of the transition, in particular, its continuity or discontinuity, is not well described within this approximation. In this work we address the important question of the phase diagram of the monoaxial chiral helimagnet in the magneticfield plane by solving the model without any uncontrolled approximation, and the results obtained are, in some respects, rather different from those in Ref. [15].

The paper is organized as follows. In Sec. II the model is described and the method of solution is outlined. Section III is devoted to analyzing the results of the phase diagram. In Sec. IV the nature of the singularities that appear on the transition line is studied. The effect of easy-plane anisotropy is discussed in Sec. V. A comparison with approximate solutions, namely, the conical approximation of Ref. [15] and the decoupling approximation developed in this work, is presented in Sec. VI, where it is also shown that the form of the phase transition line is universal when the magnitude of the anisotropy is high. Section VII deals with the application of the results to $\mathrm{CrNb}_{3} \mathrm{~S}_{6}$. And some conclusions are gathered in Sec. VIII. Finally, details on the numerical methods are given in the Appendix.

\section{MODEL AND METHOD OF SOLUTION}

To settle the question raised in Sec. I, we studied the zero-temperature ground state of a classical spin system with FM isotropic exchange, monoaxial DM interactions, and single-ion easy-plane anisotropy, in the presence of an external magnetic field.

At zero temperature the spins lying on the planes perpendicular to the DM axis are FM arranged. The competition between the DM and the FM interaction gives rise to a helicoidal spin structure along the DM axis and the model becomes effectively one-dimensional (1D). The classical effective 1D Hamiltonian in the continuum limit reads

$$
\mathcal{H}=\int_{0}^{\Lambda} d z\left[\frac{1}{2} \hat{n}^{\prime 2}-q_{0} \hat{z} \cdot\left(\hat{n} \times \hat{n}^{\prime}\right)+\gamma n_{z}^{2}-\vec{\beta} \cdot \hat{n}\right],
$$

where $z$ labels the coordinate along the DM axis $\hat{z}$, the prime stands for the derivative with respect to $z, \hat{n}$ is a unit vector in the direction of the spin $\vec{S}$, so that $\vec{S}=S \hat{n}$, with $S$ being the spin modulus, and $\Lambda$ is the system length along the DM axis, which is assumed to be large. The constants entering Eq. (1) are related to the interaction strengths as follows: let $J$ and $D$ stand for the strengths of the FM and DM interactions, respectively, $K$ for the strength of the single-ion anisotropy interaction, and $a$ for the underlying lattice parameter; the energy $\mathcal{H}$ is measured in units of $J S^{2} / a$, and therefore $q_{0}=D / J a$, $\gamma=K / J a^{2}$, and $\vec{\beta}=\left(g \mu_{\mathrm{B}} / J S a^{2}\right) \vec{H}$, where $\vec{H}$ is the applied magnetic field. The parallel magnetic field is thus proportional to $\beta_{z}$, and without any loss, we can take the perpendicular field proportional to $\beta_{x}$ and set $\beta_{y}=0$. The parameter $q_{0}$ is the helix pitch at zero magnetic field, so that its period is $L_{0}=2 \pi / q_{0}$. We set $q_{0}=1$ in the computations, which merely amounts to setting the unit length equal to $L_{0} / 2 \pi$.

At zero temperature the classical ground state is given by the minimum of the Hamiltonian, (1), which is a solution of the corresponding Euler-Lagrange (EL) equations. The infinitely many solutions are characterized by the boundary conditions (BCs). On physical grounds, we expect a periodic ground state for an infinite system. Then, if $L$ is the period, the appropriate $\mathrm{BC}$ is $\hat{n}(0)=\hat{n}(L)$. This, however, does not guarantee periodicity because, as the EL equations are second order, periodicity requires also the equality of the first derivatives,

$$
\hat{n}^{\prime}(0)=\hat{n}^{\prime}(L),
$$

a condition which cannot be generally imposed since the associated boundary value problem (BVP) would be overdetermined. Hence, the value of the spin at the boundaries, $\hat{n}(0)$, has to be tuned in order to obtain periodicity with period $L$. This can, in principle, be done, as we have the two degrees of freedom associated with $\hat{n}(0)$ and only two of the three Eqs. (2) are independent, due to the constraint $\hat{n}(z) \cdot \hat{n}^{\prime}(z)=0$. The periodic solutions are thus solely characterized by the period $L$. As the solution of a BVP is not necessarily unique, there might be several periodic solutions associated with a given $L$. We do not expect this on physical grounds, and indeed we never found more than one solution for the BVP in the course of our numerical computations. The equilibrium period is obtained by minimization of the energy density, $\mathcal{E}=\mathcal{H} / \Lambda$, which for a periodic state is given by

$$
\mathcal{E}=(1 / L) \int_{0}^{L} h(z) d z
$$

where $h(z)$ is the integrand entering Eq. (1).

The approach proposed here is a generalization of the well-known method of expansion of the magnetization in harmonic modes and selecting the mode which minimizes the free energy. The present problem, however, is highly nonlinear and all modes contribute to the solution, which has to be found by numerical techniques. Dzyaloshinskii $[5,6]$ solved analytically the case of a purely perpendicular field using a similar approach: he got the general solution of the differential equations and minimized the free energy in terms of the nontrivial integration constant, the Jacobian elliptic modulus, which is directly related to the period of the IC structure.

The form of the EL equations depends on the coordinate system chosen to parametrize $\hat{n}$. As the whole sphere cannot be smoothly parametrized with a single coordinate set, we used two different coordinate sets: (a) coordinate set I, $(\xi, \varphi)$, with $\xi \in(-\infty, \infty)$ and $\varphi \in[0,2 \pi]$, so that $\hat{n}=$ $(\cos \varphi, \sin \varphi, \xi) / \rho$, where $\rho=\sqrt{1+\xi^{2}}$; and (b) coordinate set II, $(\vartheta, \phi)$, with $\vartheta \in[0, \pi]$ and $\phi \in[0,2 \pi]$, so that $\hat{n}=$ $(\sin \vartheta \sin \phi, \cos \vartheta, \sin \vartheta \cos \phi)$. Coordinate set I is closely related to the polar coordinate set with polar axis along the DM axis $(\hat{z})$ and the polar angle $\theta$ related to $\xi$ by $\cot \theta=\xi$. This coordinate set is singular on $\pm \hat{z}$, where $\xi$ diverges. Coordinate set II is a polar coordinate system with the polar axis along $\hat{y}$ and, therefore, is singular at $\pm \hat{y}$, where $\sin \vartheta=0$. The EL equations and the BCs read, in coordinate set I,

$$
\begin{gathered}
\xi^{\prime \prime}-2 \xi \xi^{\prime 2} / \rho^{2}+\left(\varphi^{2}-2 q_{0} \varphi^{\prime}\right) \xi \\
-2 \gamma \xi-\beta_{x} \rho \xi \cos \varphi+\beta_{z} \rho=0, \\
\varphi^{\prime \prime}-2\left(\varphi^{\prime}-q_{0}\right) \xi \xi^{\prime} / \rho^{2}-\beta_{x} \rho \sin \varphi=0, \\
\varphi(0)=0, \quad \varphi(L)=2 \pi, \quad \xi(0)=\xi(L)=\xi_{0},
\end{gathered}
$$


and, in coordinate set II,

$$
\begin{gathered}
\vartheta^{\prime \prime}-\sin \vartheta \cos \vartheta \phi^{\prime 2}+2 q_{0} \sin ^{2} \vartheta \cos \phi \phi^{\prime} \\
-2 \gamma \sin \vartheta \cos \vartheta \cos ^{2} \phi+\beta_{x} \cos \vartheta \sin \phi \\
+\beta_{z} \cos \vartheta \cos \phi=0, \\
\sin ^{2} \vartheta \phi^{\prime \prime}+\sin \vartheta \cos \vartheta \vartheta^{\prime} \phi^{\prime}-2 q_{0} \sin ^{2} \vartheta \cos \phi \vartheta^{\prime} \\
+2 \gamma \sin ^{2} \vartheta \sin \phi \cos \phi+\beta_{x} \sin \vartheta \cos \phi \\
-\beta_{z} \sin \vartheta \sin \phi=0, \\
\vartheta(0)=\vartheta(L)=\pi / 2, \quad \phi(0)=\phi(L)=\phi_{0} .
\end{gathered}
$$

As $\hat{n}$ contains two degrees of freedom and the differential equations are second order, the general solution contains four independent constants. The condition $\hat{n}(0)=\hat{n}(L)$ removes two of them. We used translational invariance to eliminate one more, so that either $\xi_{0}$ or $\phi_{0}$ remained as the tunable parameter. The reason is that the configuration that minimizes the energy will have at least one point with the spin lying on the plane determined by the DM axis $(\hat{z})$ and the magnetic field $(\hat{x})$. Using translational symmetry we can choose this point as $z=0$, so that in coordinate set I we have $\varphi(0)=0$, and in coordinate set II $\vartheta(0)=\pi / 2$.

The ground-state (or equilibrium) configuration $\hat{n}(z)$ with $z \in[0, L]$ can be visualized as a closed path on the unit sphere, with the coordinate $z$ acting as the parameter of the curve. Figure 1 displays such paths, given by the red trajectories, in
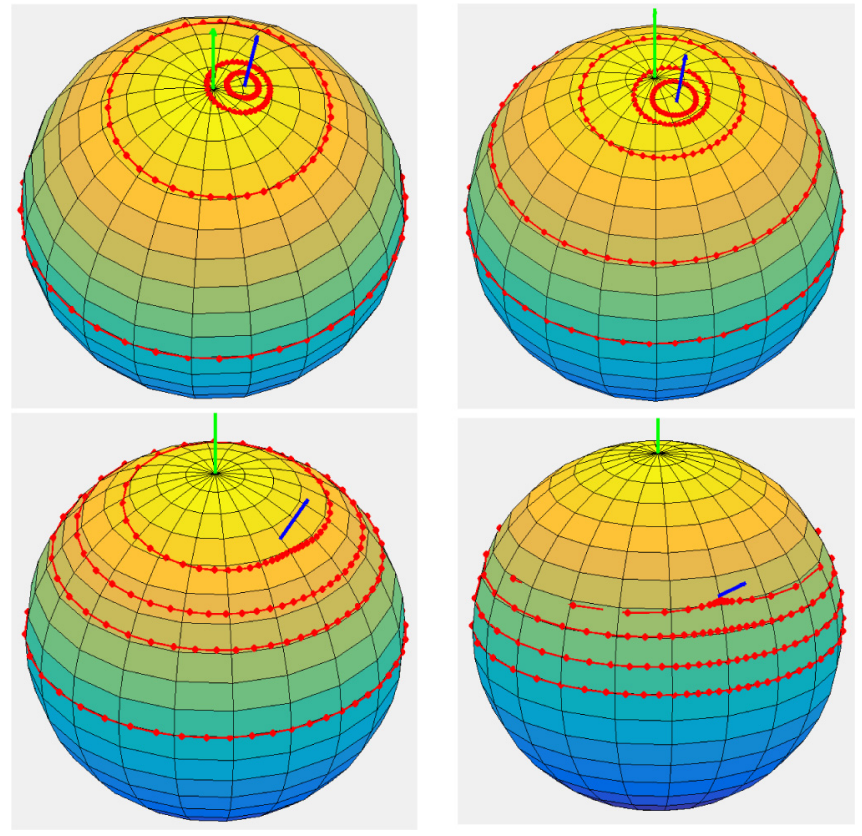

FIG. 1. Paths defined by the ground-state configurations on the unit sphera (red curves) in different typical cases: top left, $\beta_{x}=$ 0.15 (continuous transition to the FFM state); top right, $\beta_{x}=0.17$ (discontinuous transition); bottom left, $\beta_{x}=0.35$ (discontinuous transition); bottom right, $\beta_{x}=0.55$ (continuous transition). In each case different curves correspond to different values of $\beta_{z}$. The green and blue axes represent, respectively, the DM axis and the direction of the FFM state at the transition point. different situations. The green axis represents the DM axis $(\hat{z})$ and the FFM state at the transition point is represented by the blue axis. Coordinate set I and its associated BCs are appropriate when the path does encircle the DM axis; coordinate set II is suitable when the paths are not too close to $\hat{y}$, which lies on the equatorial plane. As already mentioned, note that the $\mathrm{BC},(4 \mathrm{c})$, forces the path to encircle the DM axis. When the values of the magnetic field are such that the equilibrium state is represented by one path which does not encircle the DM axis, as in the top panels in Fig. 1, the BVP, (4), has no solution, and we are forced to use coordinate set II and solve (5). On the other hand, in a wide part of the phase diagram both coordinate sets can be used. In such cases, we solved both BVPs and got the same solution $\hat{n}(z)$ within the tiny numerical uncertainties (see the Appendix, where details on the numerical procedures are given).

\section{PHASE DIAGRAM}

To obtain a phase diagram we compare the energies of the IC state, $\mathcal{E}_{\text {IC }}$, which is computed numerically following the lines outlined in the previous section, and of the FFM state, $\mathcal{E}_{\mathrm{FFM}}$, for which $\varphi=0$ and the constant value of $\xi$ is given by the solution of

$$
\frac{\xi}{\sqrt{1+\xi^{2}}}=\frac{\beta_{z}}{2 \gamma+\beta_{x} \sqrt{1+\xi^{2}}} .
$$

For small $\vec{\beta}, \mathcal{E}_{\mathrm{FFM}}>\mathcal{E}_{\mathrm{IC}}$ and the IC state is the ground state. The transition to the FFM state takes place when $\mathcal{E}_{\mathrm{FFM}}=\mathcal{E}_{\mathrm{IC}}$ and is continuous if, at this point, the IC state merges smoothly with the FFM. Otherwise the transition is discontinuous. The IC state can be visualized as a closed curve on the unit sphere, while the FFM state is represented by a single point (Fig. 1). As discussed in Sec. I, there are two mechanisms by which the IC state can be continuously transformed into the FFM state. The first possibility is displayed in the top-left panel in Fig. 1: as the magnetic field is tuned to its critical value the IC curve reduces its size until it collapses onto the FFM state. In this case a helical conical state, which, in the vicinity of the transition point, revolves around the direction of the FFM and not around the DM axis, becomes completely parallel at the transition point. The bottom-right panel in Fig. 1 illustrates the second possibility. The length of the IC curve on the unit sphere remains finite as the transition point is approached. Near the transition, the vast majority of the spins, however, are concentrated on a narrow arc close to the FFM state and the number of spins lying on the remaining part of the curve becomes negligible, so that a CSL is formed. At the transition point the period of the soliton diverges and therefore its fundamental wave vector, $q=2 \pi / L$, tends to 0 .

Figure 2 displays the phase diagram in the $\left(\beta_{x}, \beta_{z}\right)$ plane for $\gamma=2.584 q_{0}^{2}$ [Fig. 2(a)] and $\gamma=0$ [Fig. 2(b)]. We discuss the $\gamma=0$ case in Sec. V and the $\gamma=2.584 q_{0}^{2}$ case here, which is relevant for $\mathrm{CrNb}_{3} \mathrm{~S}_{6}$ (see Sec. VII). We see that the transition is continuous in the vicinities of the $\beta_{x}=0$ and the $\beta_{z}=0$ axes, while it is discontinuous in the intermediate regime. The behavior of $\mathcal{E}_{\mathrm{FFM}}-\mathcal{E}_{\mathrm{IC}}$ as a function of $\beta_{z}$ for fixed $\beta_{x}$ is illustrated in the top panels in Fig. 3 in three cases, one for each of the three transition lines. We used $\mathcal{O P}=1-M$, where $M=\left|(1 / L) \int_{0}^{L} \hat{n} d z\right|$ is the magnetization in suitable units, as 


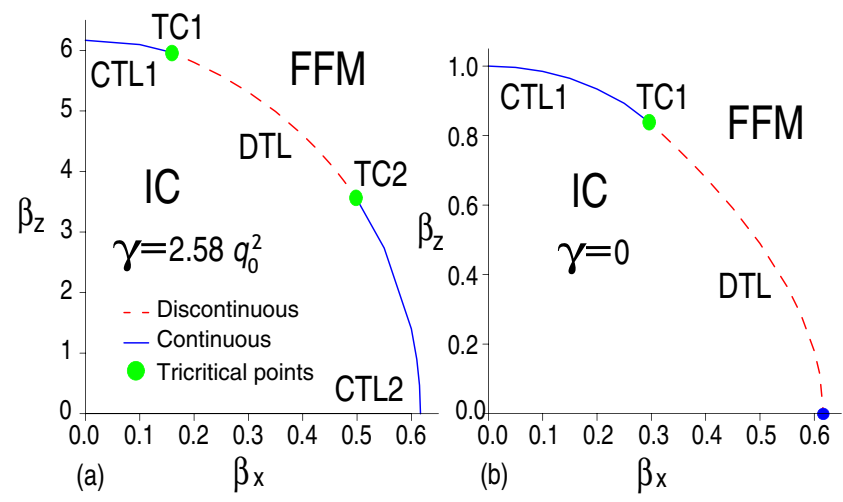

FIG. 2. Phase diagram in the $\left(\beta_{x}, \beta_{z}\right)$ plane with easy-plane anisotropy $\gamma=2.584 q_{0}^{2}$ (a) and $\gamma=0$ (b). Dashed red and solid blue lines represent, respectively, discontinuous and continuous phase transitions. Green circles show the tricritical points.

an "order parameter" (OP), which vanishes in the FFM phase. Its behavior in each case is displayed in the bottom panels in Fig. 3.

For conciseness, we call the continuous transition lines which touch the $\beta_{x}=0$ and $\beta_{z}=0$ axes, respectively, continuous transition lines 1 (CTL1) and 2 (CTL2), and we abbreviate discontinuous transition line DTL. On CTL1 the FFM state is reached continuously by the "closing cone" mechanism, as illustrated in Fig. 1 (top left). The period $L$ remains finite and close to $L_{0}$ at the transition point. On the other hand, on CTL2, the period of the IC diverges and the FFM state is continuously reached by the same mechanism as in the $\beta_{z}=0$ case. The bottom-right panel in Fig. 1 is an example of this mechanism.
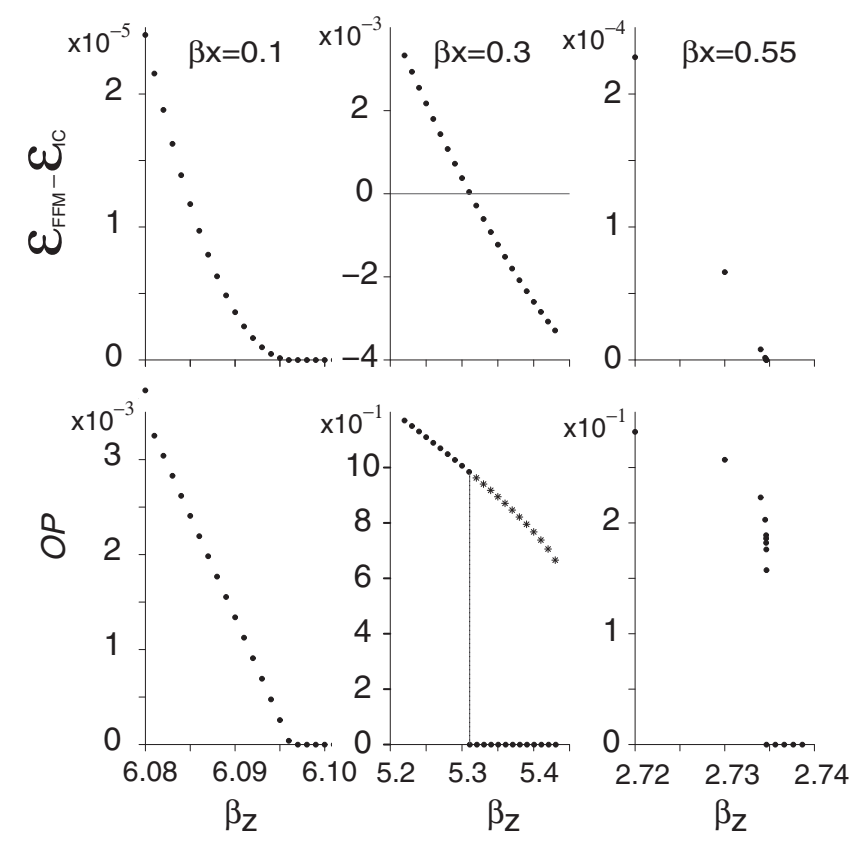

FIG. 3. $\Delta \mathcal{E}=\mathcal{E}_{\mathrm{FFM}}-\mathcal{E}_{\mathrm{IC}}$ and $\mathcal{O P}=1-M$ for three values of $\beta_{x}$, for a system with easy-plane anisotropy $\gamma=2.584 q_{0}^{2}$.
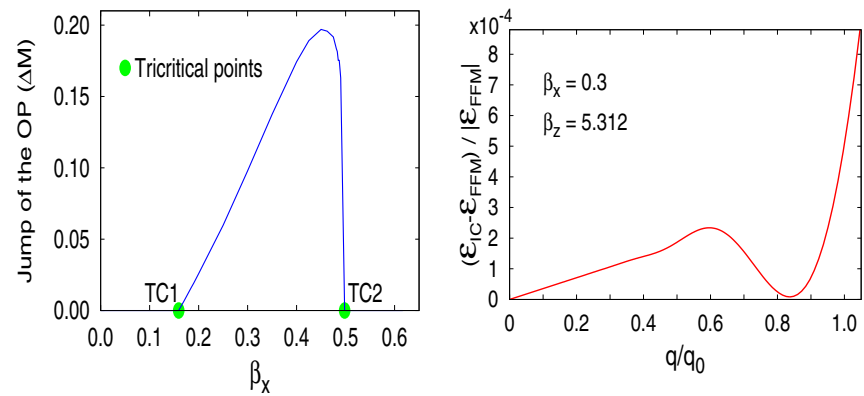

FIG. 4. Left: Order parameter jump along the transition line parametrized by $\beta_{x}$. Right: Excess of energy of the IC state over the FFM state as a function of the fundamental wave vector $q=2 \pi / L$; in this case the FFM state $(q=0)$ is stable and the IC state with $q \approx 0.84 q_{0}$ is metastable. In both figures the easy-plane anisotropy is $\gamma=2.584 q_{0}^{2}$.

On the DTL the IC and FFM state become degenerate, and the cone does not close and the period does not diverge. These situations are illustrated in the top-right and bottom-left panels in Fig. 1. The OP is discontinuous on this line. Figure 4 (left) shows the OP jump along the transition line, parametrized by $\beta_{x}$. Along the DTL the IC state coexists with the FFM state, as they are degenerate in energy. On each side of the line either the IC or the FFM state is metastable. This is illustrated in Fig. 4 (right), where the energy difference is displayed as a function of $q=2 \pi / L$ in a case in which the IC state with $q \approx 0.84 q_{0}$ is metastable and the FFM $(q=0)$ is stable. Hence, phenomena like phase coexistence, domain formation, and hysteresis are expected in some regions of the magnetic phase diagram. The metastability does not cause numerical problems since $L$ is fixed in the numerical computations, and the BCs, (4c), prevent switching between the IC and the FFM states.

The DTL is separated from CTL1 and CTL2 by two tricritical points, called, respectively, TC1 and TC2. In Fig. 4 (left) these are the points where the OP jump ceases to vanish, and they are represented by the two green circles. For $\gamma=2.584 q_{0}^{2}$ TC1 is located at $\left(\beta_{x}^{t 1}, \beta_{z}^{t 1}\right)=(0.1593,5.9552)$ and TC2 at $\left(\beta_{x}^{t 2}, \beta_{z}^{t 2}\right)=(0.498,3.5301)$.

Figure 5 displays the fundamental wave vector $q$ as a function of $\beta_{z}$ for different values of $\beta_{x}$, including $\beta_{x}^{t 1}=0.159$ and $\beta_{x}^{t 2}=0.498$ (dashed lines with black symbols). Note that $q$ is almost constant $\left(q \approx q_{0}\right)$ for $\beta_{x}<\beta_{x}^{t 1}$, while it decreases with $\beta_{x}$ and with $\beta_{z}$ for $\beta_{x}>\beta_{x}^{t 1}$. The variation of $q$ becomes very abrupt close to the tricritical point TC2.

The spatial dependence of the ground state as the transition is approached is shown in Fig. 6, where the Cartesian components of $\hat{n}$ are displayed as a function of $z / L$. Cartesian components are shown since they are regular for any $\vec{\beta}$. They have been computed from the solutions obtained with either coordinate set I or coordinate set II. The left panels correspond to $\beta_{x}=0.15$ and show $\hat{n}(z)$ for different values of $\beta_{z}$ which tend to a transition point on CTL1. We observe that the variation of $\hat{n}(z)$ is distributed smoothly over the whole period for all $\beta_{z}$, so that no soliton is formed. Also, the amplitude of the oscillations decreases smoothly to 0 as the transition point is approached, continuously attaining the FFM. The 


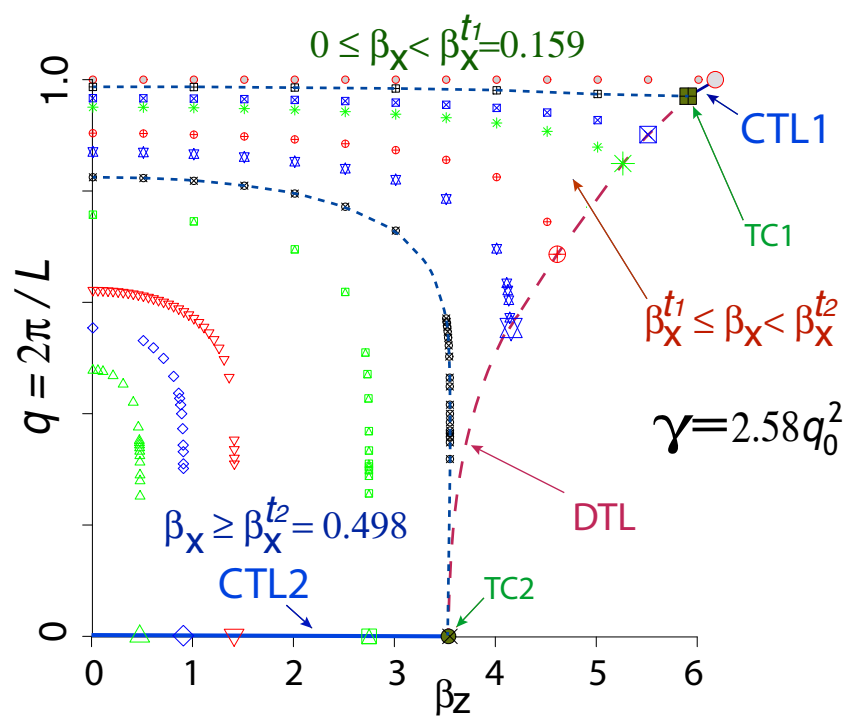

FIG. 5. Inverse of the period of the IC state as a function of $\beta_{z}$ for several values of $\beta_{x}$. The critical $\beta_{z}$ for each $\beta_{x}$ is given by the point with minimum $q$ in the corresponding curve. The two tricritical points are given by the points with minimum $q$ on each of the two dashed lines $\left(\beta_{x}^{t 1}=0.159\right.$ and $\left.\beta_{x}^{t 2}=0.498\right)$ with black symbols.

right panels correspond to $\beta_{x}=0.55$ and the values of $\beta_{z}$ tend to a transition point on CTL2. Observe that the variations of $\hat{n}(z)$ are gradually concentrated in a narrow section of the period, and a soliton lattice is formed. It is a relative
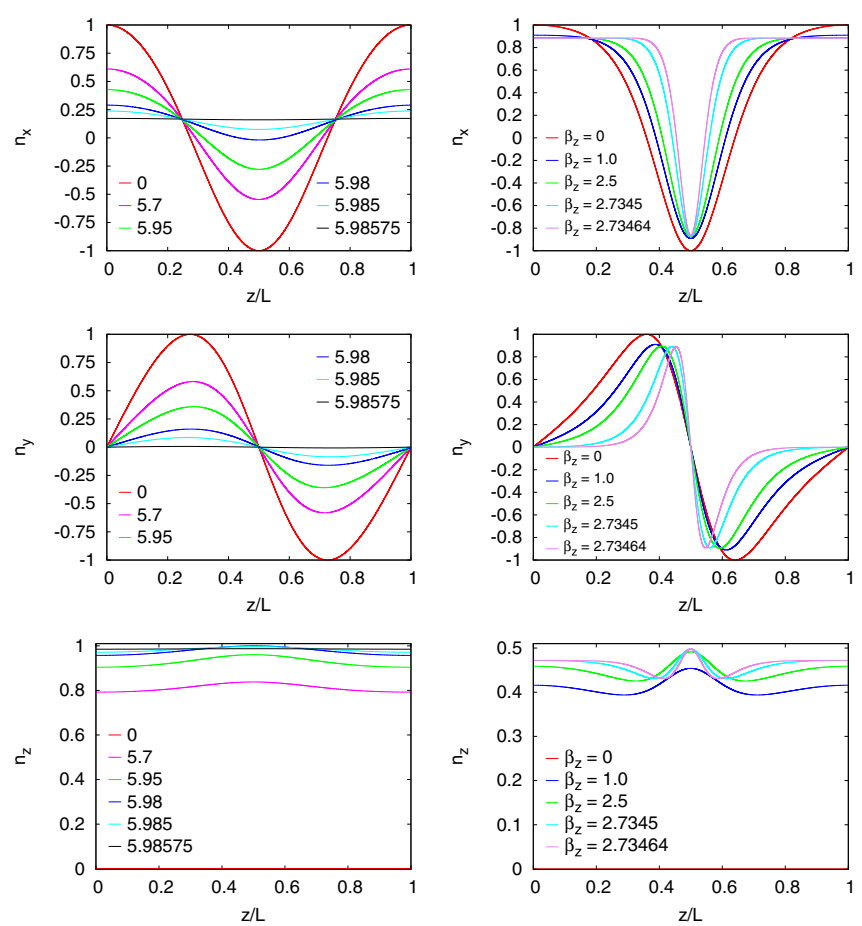

FIG. 6. Cartesian components of $\hat{n}$ as a function of $z / L: n_{x}$ (top), $n_{y}$ (middle), and $n_{z}$ (bottom), for $\gamma=2.584 q_{0}^{2}, \beta_{x}=0.15$ (left) and $\beta_{x}=0.55$ (right), and different values of $\beta_{z}$ approaching the transition point. The DM axis and the magnetic field point in the direction of $\hat{z}$ and $\hat{x}$, respectively. narrowness: the size of the region where $\hat{n}(z)$ varies noticeably is approximately constant, but the period increases as the transition is approached. It is interesting that the soliton lattice can be created by increasing the component of the magnetic field parallel to the DM axis, keeping the perpendicular field constant.

\section{SINGULAR BEHAVIOR ON THE CONTINUOUS TRANSITION LINES}

On the continuous transition lines CTL1 and CTL2 the IC and FFM states merge continuously but, as in any phase transition, physical observables may present peculiar singularities. Let us analyze the nature of these singularities. The OP vanishes linearly as the CTL1 $\left(\beta_{x}<\beta_{x}^{t 1}\right)$ is attained from the IC phase (bottom left panel in Fig. 3). Thus, no singularity appears on CTL1. As TC1 is approached, the slope of the OP becomes more abrupt and becomes singular at TC1. We assume that at TC1 the OP vanishes as a power law, $\mathcal{O P} \sim A\left(\beta_{z}^{t 1}-\beta_{z}\right)^{n / m}$, with $n$ and $m$ small integers. The best fit, which is indeed very good, gives $\beta_{z}^{t 1} \approx 5.9552506$ and $n / m=0.5002 \approx 1 / 2$.

As CTL2 is attained from the IC phase, the OP vanishes more abruptly than a power (bottom right panel in Fig. 3). The OP scales with the period as $1 / L$, so that the OP singularity is directly related to the $L$ singularity. For $\beta_{z}=0$ the following scaling law is derived from the analytically known solution [6]:

$$
\left(\sqrt{\beta_{x c}} L+1\right) \exp \left(-\sqrt{\beta_{x c}} L\right) \sim\left(\beta_{x c}-\beta_{x}\right) / 8 \beta_{x c},
$$

where $\beta_{x c}=\left(\pi^{2} / 16\right) q_{0}^{2}$ is the critical field at $\beta_{z}=0$. Along CTL2, $\left(\beta_{x}^{t 2}<\beta_{x} \leqslant \beta_{x c}\right)$, a generalization of the above scaling law, given by

$$
B(A L+1) \exp (-A L) \sim\left(\beta_{c}-\beta\right) / 8 \beta_{c},
$$

holds. In this expression $\beta$ stands either for $\beta_{x}$ or for $\beta_{z}$, and the other component of $\vec{\beta}$ has to be kept constant. The parameters $A$ and $B$ vary smoothly along CTL2. The value of $B$ depends on whether CTL2 is attained keeping $\beta_{x}$ or $\beta_{z}$ constant, but $A$, which characterizes the singularity, does not. Figure 7 displays an example of the scaling of $L$ as the transition point

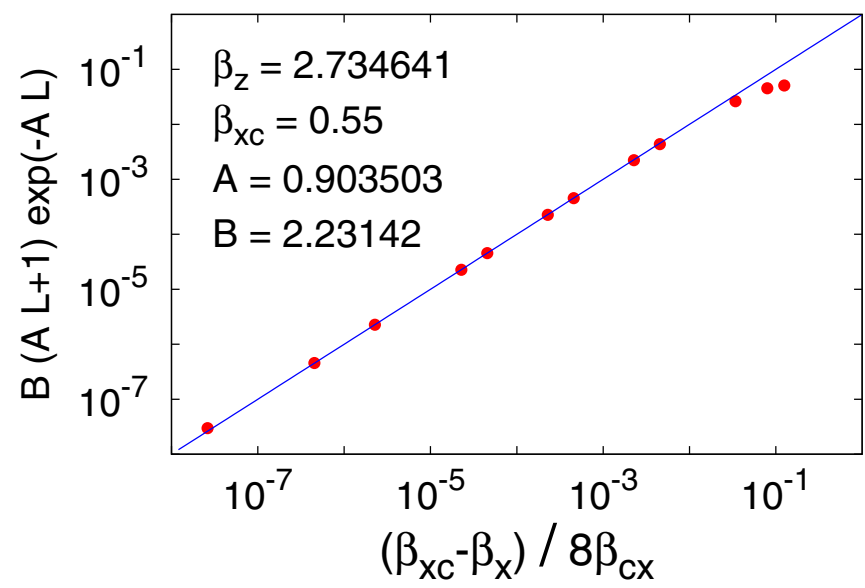

FIG. 7. Scaling of the period $(L)$ as CTL2 is approached along the $\beta_{z}=2.734641$ line for $\gamma=2.584 q_{0}^{2}$. The blue line is $y=x$ on an $(x, y)$ plane. 
$\left(\beta_{x}=0.55, \beta_{z}=2.734641\right)$ is approached along the line of constant $\beta_{z}$. The parameter $A$ grows as $\beta_{x}$ decreases and diverges at the tricritical point TC2. This means that the nature of the singularity changes at the tricritical point. Unfortunately, this new singularity cannot be studied numerically without further insight.

The scaling of $L$ given by (8) is a general feature of the formation of the CSL, and it can be used to fit the experimental results near the transition point for oblique fields. The behavior of the parameter $A$ then can be used to locate the tricritical point TC2.

\section{EFFECT OF THE EASY-PLANE SINGLE-ION ANISOTROPY}

The easy-plane anisotropy has an important effect on the phase diagram, as shown in Fig. 2(b), where the $\gamma=0$ case is displayed. We see that the CTL2 disappears, the DTL reaches the $\beta_{z}=0$ axis, and only one tricritical point (TC1) appears. For $\gamma>0$ the phase diagram is qualitatively similar to the case discussed in the previous sections $\left(\gamma=2.584 q_{0}^{2}\right)$. The transition lines in the $\left(\beta_{x}, \beta_{z}\right)$ plane for different values of $\gamma$ are shown in Fig. 8. By increasing $\gamma$ the lengths of CTL1 and CTL2, respectively, decrease and increase. In a tridimensional parameter space $\left(\beta_{x}, \beta_{z}, \gamma\right)$ there is a phase transition surface divided by two tricritical lines into three sectors, one in which the transitions are discontinuous and two in which they are continuous.

Not surprisingly, the easy-plane anisotropy provides stability to the CSL formed when the field is purely perpendicular, so that for large $\gamma$ large parallel fields are necessary to modify the behavior of the CSL and its transition to the FFM state. The effect of easy-plane anisotropy is important since low-anisotropy compounds described by the model studied in this work, as, for instance, thin films of MnSi [16], will be characterized by low values of $\gamma$.

The case of easy-axis aniostropy $(\gamma<0)$, not analyzed in this work, is theoretically interesting-and perhaps also phenomenologically - as peculiar phenomena may take place in the crossover to Ising-like behavior as $|\gamma|$ increases [17].

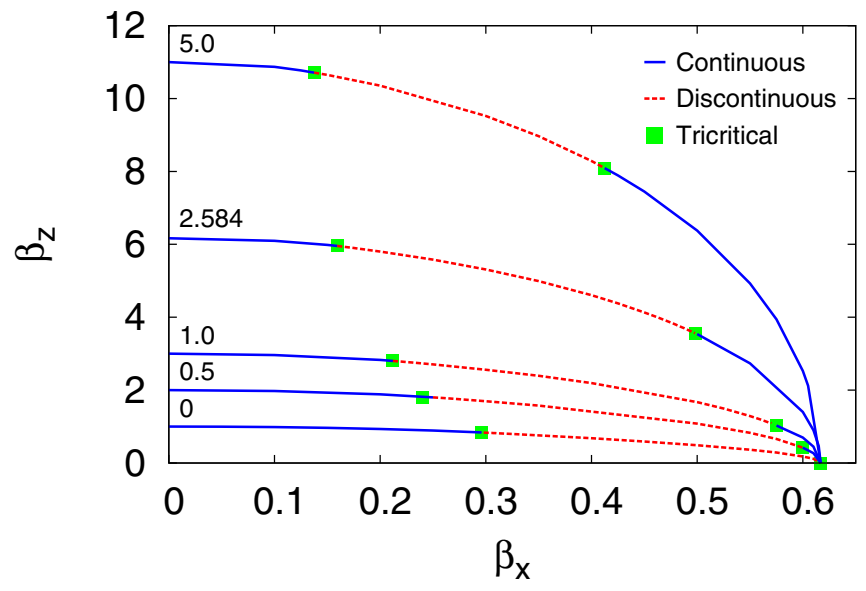

FIG. 8. Transition lines in the $\left(\beta_{x}, \beta_{z}\right)$ plane for different values of the easy-plane single-ion anisotropy $\gamma / q_{0}^{2}$.

\section{COMPARISON WITH SIMPLIFYING APPROXIMATIONS}

It is interesting to compare the results obtained here with those obtained by making approximations which drastically simplify the mathematical problem. We consider two such approximations: the conical approximation from Ref. [15] and the decoupling approximation developed below.

In the conical approximation the spatial variation of the spin component parallel to the DM axis, $n_{z}$, is neglected, and the ground state is obtained by minimizing the energy density over spin configurations with constant $n_{z}=\cos \theta_{0}$. The EL equations for the nonconstant components give a sine-Gordon equation similar to that of the $\beta_{z}=0$ model with an effective perpendicular field $\beta_{x}^{\text {eff }}=\beta_{x} / \sin \theta_{0}$. Then the ground state has the form [15]

$$
\begin{gathered}
n_{x}(z)=\sin \theta_{0} \cos \varphi(z), \\
n_{y}(z)=\sin \theta_{0} \sin \varphi(z), \\
n_{z}(z)=\cos \theta_{0},
\end{gathered}
$$

where

$$
\varphi(z)=-2 \operatorname{am}\left(\sqrt{\beta_{x} /\left(\kappa^{2} \sin \theta_{0}\right)} z, \kappa\right),
$$

and $\operatorname{am}(x, k)$ is the Jacobi amplitude function with elliptic modulus $\kappa$. The energy density associated with the above solution depends on the two parameters $\cos \theta_{0}$ and $\kappa$ and reads [15]

$$
\begin{aligned}
\mathcal{E}= & \gamma \cos ^{2} \theta_{0}-\beta_{z} \cos \theta_{0}-\frac{q_{0}^{2}}{2} \sin ^{2} \theta_{0}-\beta_{x} \sin \theta_{0} \\
& \times\left(\frac{2}{\kappa^{2}}+\frac{\pi \sqrt{q_{0}^{2} \sin \theta_{0} / \beta_{x}}}{\kappa K(\kappa)}-\frac{4 E(\kappa)}{\kappa^{2} K(\kappa)}-1-\frac{q_{0}^{2}}{2 \beta_{x}} \sin \theta_{0}\right),
\end{aligned}
$$

where $K(\kappa)$ and $E(\kappa)$ are the complete elliptic integrals of the first and second kind, respectively. The first three terms give the energy of the conical helix with wave vector $q_{0}$, while the term proportional to $\beta_{x}$ is the difference between the conical helicoid, (12), and the conical helix. We write the energy in this way for later convenience.

Minimization of (13) with respect to $\theta_{0}$ and $\kappa$ gives the phase diagram. The IC-FFM transition is everywhere discontinuous except at the two end points of the transition line. Note that the conical approximation is exact in the two limiting cases $\beta_{x}=0$ and $\beta_{z}=0$. The goodness of the approximation depends on the magnitude of the easy-plane anisotropy. Figure 9 displays the phase transition lines obtained in this work by numerical integration of the EL equations (EL solution) and, in the conical approximation, for $\gamma=0$ (top) and $\gamma=2.584$ (bottom). In the $\gamma=0$ case the transition line in the conical approximation deviates considerably from the line given by the EL solution. However, for $\gamma=2.584$ the conical approximation predicts a transition line which is very close to the EL solution line. As the energy of the approximate conical helicoid is always higher than the energy of the ground state the approximate transition line lies always below the EL solution transition line, as can be appreciated in Fig. 9. 

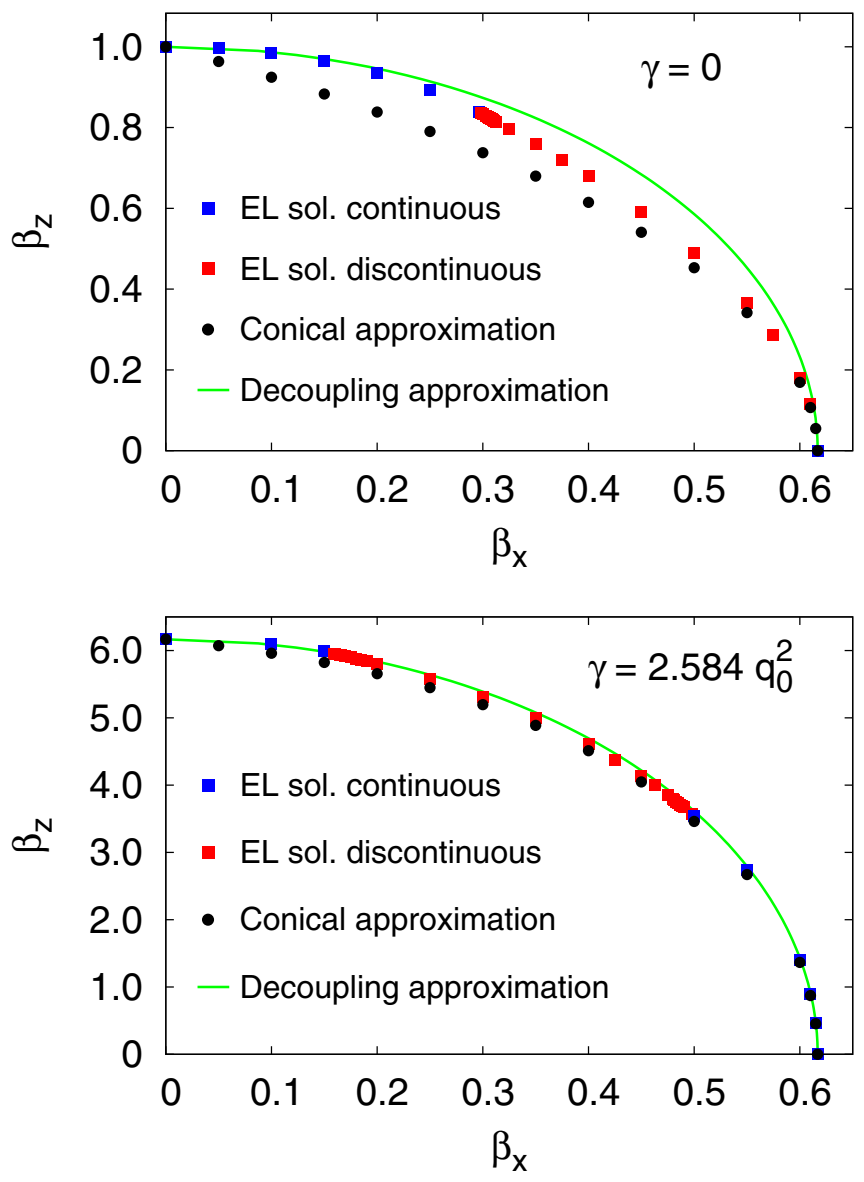

FIG. 9. The phase transition line obtained in the conical approximation in Ref. [15] (filled black circles) and in the decoupling approximation in this work (solid green line) compared with the result of the numerical solution of the Euler-Lagrange equations, for easy-plane single-ion anisotropy $\gamma=0$ (top) and $\gamma=2.584$ (bottom).

It is easy to understand why the conical approximation locates the transition line very accurately as the magnitude of the easy-plane anisotropy increases. The energy $\mathcal{H}$ given by Eq. (1) contains a term $\gamma n_{z}^{2}-\beta_{z} n_{z}=\gamma\left(n_{z}-\beta_{z} / 2 \gamma\right)^{2}-$ $\beta_{z}^{2} / 4 \gamma$. If $\gamma$ is very large, the energy penalty caused by a large deviation of $n_{z}$ from the constant value $\beta_{z} / 2 \gamma$ cannot be compensated by a gain provided by the other terms in $\mathcal{H}$. In the vicinity of the transition, however, the energy of the IC state is only slightly lower than the FFM energy and the conical approximation does not capture the nature of the transition. The differences between the IC and the FFM states are precisely due to the spatial variation of the spin. The small variation of $n_{z}$ allows the IC to reach the FFM state continuously. Consider, for instance, the transition at constant $\beta_{x}$ depicted in the top-left panel in Fig. 1. The transition takes place continuously when the spin paths (red points) collapse onto the FMM (blue axis). This is not possible in the conical approximation, in which the spin configuration paths are forced to follow the parallels of the unit spheres in Fig. 1. As it gives discontinuous transitions along the whole transition line, the conical approximation does not predict the continuous transitions or the tricritical behavior.
Let us develop a further approximation, which we call the decoupling approximation, in which we assume also a conical ground state. We denote by $\beta_{x c}=\left(\pi^{2} / 16\right) q_{0}^{2}$ and $\beta_{z c}=2 \gamma+q_{0}^{2}$ the parallel and perpendicular critical fields, respectively. We note that, for large $\gamma$, the value of $\cos \theta_{0}$ is determined basically by the three first terms in (13). The term proportional to $\beta_{x}$, which, we recall, is the difference between the energies of the conical helicoid, (12), and the conical helix with $\varphi(z)=q_{0} z$, gives a minor contribution. Indeed, at low $\beta_{x}$ and $\beta_{z}$ the conical helicoid is a slightly distorted conical helix; at low $\beta_{x}$ and large $\beta_{z}$ the term proportional to $\beta_{x}$ is clearly a small correction; and for $\beta_{x}$ close to $\beta_{x c}$ the energy of the conical helicoid is very close to the energy of the FFM state, which happens to be close to the energy of the conical helix [18]. Hence, in the decoupling approximation we have $\cos \theta_{0}=\beta_{z} / \beta_{z c}$. Minimization of the energy with respect to $\kappa$ gives an equation similar to that in the well-known $\beta_{z}=0$ case: $E(\kappa) / \kappa=\sqrt{\sin \theta_{0} \beta_{x c} / \beta_{x}}$. The phase transition takes place continuously when $\kappa=1$, that is, when $\beta_{x} / \beta_{x c}=\sin \theta_{0}$, and taking into account the value of $\sin \theta_{0}$, we get the following expression for the transition line:

$$
\beta_{z} / \beta_{z c}=\sqrt{1-\beta_{x}^{2} / \beta_{x c}^{2}} .
$$

The solid green lines in Fig. 9 are the curves corresponding to the above equation. We see that they describe very accurately the transition line for high easy-plane anisotropy, but it departs notably from the EL solution for low $\gamma$. Interestingly, Eq. (14) shows that the form of the transition line for high anisotropy is universal when expressed in terms of the dimensionless fields $\beta_{x} / \beta_{x c}$ and $\beta_{z} / \beta_{z c}$. We see that the decoupling approximation is as accurate as the conical approximation, or even more, and it has the virtue of providing a simple analytic expression for the transition line. On the other hand, the decoupling approximation suffers from the same limitations as the conical approximation and does not predict correctly the nature of the transition, as it always gives continuous transitions.

\section{APPLICATION TO $\mathrm{CrNb}_{3} \mathrm{~S}_{6}$}

The general results obtained in this work can be readily applied to $\mathrm{CrNb}_{3} \mathrm{~S}_{6}$. For this compound, $a \approx 1.2 \mathrm{~nm}$. Other measurable parameters like the critical parallel and perpendicular fields, $H_{x c}$ and $H_{z c}$, respectively, and the the zero-field helix period, $L_{0}$, depend strongly on the sample. As an example [8], we take $L_{0} \approx 48 \mathrm{~nm}$ and $H_{x c} \approx 2300$ Oe. A value of $\gamma=2.584 q_{0}^{2}$ ensures the relation $H_{z c} \approx 10 H_{x c}$ typically observed experimentally. The high easy-plane anisotropy implies that the location transition line is accurately given by the results of the conical approximation in Ref. [15], and moreover, it is given by Eq. (14) derived in the present work, so that

$$
H_{z} / H_{z c}=\sqrt{1-H_{x}^{2} / H_{x c}^{2}} .
$$

The critical fields $H_{x c}$ and $H_{z c}$ depend strongly on the sample. Due to impurities, crystalline defects, etc., each sample will be theoretically described by a different set of the model effective parameters. The anisotropy, however, is expected to be high for all samples, so that Eq. (15) holds for any sample, and thus the phase transition line in $\mathrm{CrNb}_{3} \mathrm{~S}_{6}$ is universal (independent 
of the sample) when expressed in terms of the dimensionless fields $H_{x} / H_{x c}$ and $H_{z} / H_{z c}$.

The position of the tricritical points on the transition line, however, is not universal and depends on the sample. For the values reported in Ref. [8] $\left(H_{x c} \approx 2300 \mathrm{Oe}\right.$ and $H_{z c} \approx 23000 \mathrm{Oe}$ ) we get $\vec{H}=3729 \vec{\beta}$ Oe, and thus the tricritical points TC1 and TC2 are predicted to be at, respectively, $\left(H_{x}^{t 1}, H_{z}^{t 1}\right) \approx(590,22200)$ Oe and $\left(H_{x}^{t 2}, H_{z}^{t 2}\right) \approx$ (1860,12490) Oe. A CSL can be formed by approaching the CTL2, increasing the perpendicular field $H_{x}$ with the parallel field $H_{z}$ held constant below $12500 \mathrm{Oe}$, or increasing the parallel field with the perpendicular field held constant above 1850 Oe, or increasing a field tilted from the DM by an angle $\alpha$ of less than $81^{\circ}$.

Furthermore, a line of discontinuous transitions appears in the phase diagram of $\mathrm{CrNb}_{3} \mathrm{~S}_{6}$, and therefore phenomena typical of first-order transitions, like phase coexistence and hysteresis in magnetization and magnetoresistance, are expected.

\section{CONCLUSIONS}

In this work the important and long-standing problem of the determination of the phase diagram of a monoaxial chiral helimagnet at zero temperature in the magnetic-field plane has been addressed. The transition line from the incommensurate phase to the commensurate forced FM state has been thoroughly analyzed as a function of the single-ion aniotropy and the nature of the transition has been elucidated. We have shown that generically the transition line is composed of a line of discontinuous transition separated from two lines of continuous transitions by two tricritical points. The nature of the singularities at the transition line has also been thoroughly analyzed and we showed that the transition from the chiral soliton lattice to the forced FM state along the continuous transition line is characterized by logarithmic singularities analogous to those characteristic in the case of a purely perpendicular magnetic field. This characteristic singular behavior may be used to locate one of the tricritical points.

For high anisotropy the conical and the decoupling approximations give the location of the transition line very accurately, but they fail in characterizing the nature of the transition, since the former predicts a discontinuous transition along the whole line, and the latter continuous transitions everywhere. Consequently, the tricritical behavior is not predicted by any of these approximations. For high anisotropy we found that the form of the transition line is universal, given by Eq. (14) or (15). The position of the tricritical points on the transition line is not universal, however, and has to be computed for each sample.

Hence, unexpected predictions for the low-temperature regime of $\mathrm{CrNb}_{3} \mathrm{~S}_{6}$ are given: discontinuous transitions, tricritical behavior, and universality (that is, independence of the sample) of the form of the transition line due to the high anisotropy characteristic of this compound. These phenomena may have interesting applications in spintronics. It remains to study the very interesting question of how the phase diagram evolves with increasing temperature. Work in this direction is in progress [19].

\section{ACKNOWLEDGMENTS}

J.C. and V.L. acknowledge Grant No. MAT2015-68200C2-2-P from the Spanish Ministry of Economy and Competitiveness. The work of A. S. Ovchinnikov was supported by the Government of the Russian Federation Program (02.A03.21.0006) and by the Ministry of Education and Science of the Russian Federation (Project Nos. 1437 and 2725).

\section{APPENDIX: NUMERICAL PROCEDURE}

The strategy to find a solution to the problem is as follows: for given $L$ and $\xi_{0}$ or $\phi_{0}$, we solve numerically the BVP defined by either Eq. (4) or Eq. (5); for fixed $L$, we tune either $\xi_{0}$ or $\phi_{0}$ and repeat the computation until periodicity is reached, then we compute the energy per period $\mathcal{E}$ via a numerical quadrature algorithm. Finally, we determine the period $L$ which minimizes $\mathcal{E}$ with a minimization algorithm. Alternatively, we can minimize $\mathcal{E}$ as a function of the two variables $\xi_{0}$ (or $\phi_{0}$ ) and $L$. Both approaches give the same result, as the minimum of the energy as a function of the BCs for fixed $L$ is the periodic solution which satisfies Eq. (2).

To solve numerically the BVPs defined by Eq. (4) or (5) we used a relaxation method as described in Ref. [20] (see also Ref. [21]). The BVP solver works as follows. First, the two second-order differential equations are converted in a set of four first-order differential equations in the usual way, by introducing two new variables, $\omega$ and $v$, and two new equations which relate them to the derivatives of $\varphi$ and $\xi, \omega=d \varphi / d z$ and $v=d \xi / d z$. Then this system of first-order differential equations is converted in a system of finite-difference equations by substituting the derivatives by forward finite differences. Therefore, a mesh with $N+1$ points $\left(z_{0}=0, z_{1}, \ldots, z_{N}=L\right)$ is introduced. For simplicity, we used a regular mesh, so that $z_{n}-z_{n-1}=L / N$ for $n=$ $1, \ldots, N$. The finite-difference equations and the $\mathrm{BCs}$ form a set of $4(N+1)$ nonlinear algebraic equations $(4 N$ of them given by the difference equations and the remaining four provided by the BCs) with $4(N+1)$ unknowns, which are the values of $\xi(z), \varphi(z), v(z)$, and $\omega(z)$ at the mesh points. The algebraic equations are solved numerically with a Newton algorithm, which proceeds iteratively starting from one initial guess, which has to be supplied. Subsequently, $\mathcal{E}$ is computed with a simple quadrature algorithm using the values of $h(z)$ calculated at the mesh points.

Periodicity is enforced by tuning $\xi_{0}$ until the conditions $\omega(L)=\omega(0)$ and $v(L)=v(0)$ are met. The two equations can be enforced by tuning only one variable, $\xi_{0}$. The reason is that we exploited translational symmetry to eliminate the freedom in the BCs for $\varphi$, which in general should read $\varphi(0)=\varphi_{0}$ and $\varphi(L)=\varphi_{0}+2 \pi$. Hence, periodicity can be enforced by tuning $\varphi_{0}$ and $\xi_{0}$. Translational invariance implies that we can set $\varphi_{0}=0$, taking into account that, on physical grounds, there has to be a point at which the spin lies in the plane formed by the DM axis and the magnetic field (i.e., with $\varphi=0$ ). Hence, we enforce the condition $v(L)-v(0)=0$ by tuning $\xi_{0}$ via a simple bracketing-bisection algorithm, and the other condition is automatically satisfied. Finally, the minimum of $\mathcal{E}$ as a function of $L$ is obtained also with a simple bracketingbisection algorithm. 
To control the numerical errors we set very demanding values for the convergence tolerances of the different algorithms: $10^{-14}$ for the BPS solver, $10^{-12}$ for the computation of $\mathcal{E}$, and $10^{-11}$ for the bracketing-bisection algorithms. The effect of the discretization was taken into account when computing $\mathcal{E}$ via the quadrature algorithm. The mesh was refined iteratively, the number of points being doubled at each iteration, until the difference between the values of $\mathcal{E}$ computed in two successive iterations was smaller than the tolerance $\left(10^{-12}\right)$. In this way we were able to get the ground state with a high accuracy.
The main issue is the convergence of the BVP solver, which works iteratively so that to get convergence the initial guess functions have to be close enough to the actual solution. We achieve this by varying $\beta_{z}$ by small steps and using the solution found at one $\beta_{z}$ as the seed for the next step. We start at $\beta_{z}=0$, taking advantage of the fact that the solution is analytically known there. This procedure is sound, as we expect the IC structure to be continuous on $\vec{\beta}$, as it is in the cases where the field is either perpendicular or parallel. The results confirm this point.
[1] J.-i. Kishine and A. S. Ovchinnikov, Solid State Phys. 66, 1 (2015).

[2] A. Fert, V. Cross, and J. Sampaio, Nature Nanotechnol. 8, 152 (2013).

[3] N. Romming, C. Hanneken, M. Menzel, J. Bickel, B. Wolter, K. von Bergmann, A. Kubetzka, and R. Wiesendanger, Science 341, 636 (2013).

[4] T. Nagamiya, Solid State Phys. 20, 305 (1968).

[5] I. Dzyaloshinskii, J. Phys. Chem. Solids 4, 241 (1958).

[6] I. E. Dzyaloshinskii, Zh. Eksp. Teor. Fiz. 46, 1420 (1964) [Sov. Phys. JETP 19, 960 (1964)].

[7] Y. Izyumov, Sov. Phys. Usp. 27, 845 (1984).

[8] Y. Togawa, T. Koyama, T. Takayanagi, S. Mori, Y. Kousaka, J. Akimitsu, S. Nishihara, K. Inoue, A. Ovchinnikov, and J. Kishine, Phys. Rev. Lett. 108, 107202 (2012).

[9] J.-i. Kishine, I. G. Bostrem, A. S. Ovchinnikov, and V. E. Sinitsyn, Phys. Rev. B 86, 214426 (2012).

[10] A. Ovchinnikov, V. Sinitsyn, I. Bostrem, and J. Kishine, J. Exp. Theor. Phys. 116, 791 (2013).

[11] J.-i. Kishine, I. V. Proskurin, and A. S. Ovchinnikov, Phys. Rev. Lett. 107, 017205 (2011).

[12] Y. Togawa, Y. Kousaka, S. Nishihara, K. Inoue, J. Akimitsu, A. S. Ovchinnikov, and J. Kishine, Phys. Rev. Lett. 111, 197204 (2013).
[13] N. J. Ghimire, M. A. McGuire, D. S. Parker, B. Sipos, S. Tang, J.-Q. Yan, B. C. Sales, and D. Mandrus, Phys. Rev. B 87, 104403 (2013).

[14] A. C. Bornstein, B. J. Chapman, N. J. Ghimire, D. G Mandrus, D. S. Parker, and M. Lee, Phys. Rev. B 91, 184401 (2015).

[15] B. Chapman, A. Bornstein, N. Ghimire, D. Mandrus, and M. Lee, Appl. Phys. Lett. 105, 072405 (2014).

[16] M. N. Wilson, E. A. Karhu, D. P. Lake, A. S. Quigley, S. Meynell, A. N. Bogdanov, H. Fritzsche, U. K. Rößler, and T. L. Monchesky, Phys. Rev. B 88, 214420 (2013).

[17] L. Liu, Phys. Rev. Lett. 31, 459 (1973).

[18] This is no coincidence, as the phase transition takes place roughly when the energy of the helix is similar to the energy of the FFM state. Indeed, for $\beta_{z}=0$ we have that the difference between these two energies at the critical point is $-\beta_{x c}+q_{0}^{2} / 2=$ $\left(\pi^{2} / 8-1\right) q_{0}^{2} / 2 \approx 0.117 q_{0}^{2}$.

[19] V. Laliena, J. Campo, Y. Kousaka, and K. Inoue, arXiv:1603.06362 (2016).

[20] W. Press, S. Teukolsky, W. Vetterling, and B. Flannery, Numerical Recipes in $C$ (Cambridge University Press, New York, 2002).

[21] P. Eggleton, Mon. Not. R. Astron. Soc. 151, 351 (1971). 